\title{
Time delay in valence-shell photoionization of noble-gas atoms
}

\author{
A. S. Kheifets* \\ Research School of Physical Sciences, Australian National University, Canberra, Australian Capital Territory 0200, Australia
}

(Received 19 April 2013; published 6 June 2013)

\begin{abstract}
We use the nonrelativistic random-phase approximation with exchange to perform calculations of valence-shell photoionization of $\mathrm{Ne}, \mathrm{Ar}, \mathrm{Kr}$, and Xe from their respective thresholds to photon energy of $200 \mathrm{eV}$. The energy derivative of the complex phase of the photoionization matrix elements is converted to the photoelectron group delay that can be measured in attosecond streaking or two-photon sideband interference experiments. Comparison with reported time-delay measurements in $\mathrm{Ne}$ and $\mathrm{Ar}$ at a few selected photon energies is made. Systematic mapping of time delay across a wide range of photon energies in several atomic targets allows to highlight important aspects of fundamental atomic physics that can be probed by attosecond-time-delay measurements.
\end{abstract}

DOI: 10.1103/PhysRevA.87.063404

PACS number(s): 32.80.Fb, 32.30.Rj, 32.70.-n, 31.15.ve

\section{INTRODUCTION}

Time delay in atomic photoionization has become an active and rapidly expanding field of research following pioneering experiments on attosecond streaking [1] and twophoton sideband interference [2]. Both techniques use the extreme ultraviolet (xuv) pump pulse to ionize the target atom and the ir probe to obtain the timing information on the photoemission process. In attosecond streaking, the varying time delay between the pump and the probe pulses is mapped onto the photoelectron kinetic energy. The whole valence shell is projected onto a photoelectron kinetic energy map (the so-called spectrogram), which is then modeled, in the strong field or Coulomb-Volkov approximations, with the photoionization time delay being treated as a fitting parameter. This measurement revealed a relative time delay of $21 \pm 5$ as between photoemission from the $2 p$ and $2 s$ subshells in $\mathrm{Ne}$ at $106-\mathrm{eV}$ photon energy. The positive sign of the relative time delay indicates that emission of the photoelectron from the $2 p$ subshell is seemingly delayed relative to that from the $2 s$ subshell.

In the two-photon interferometric technique, the varying time delay between the pump and probe pulses is mapped onto the two-photon sideband (SB) oscillations. The phase of these oscillations depends on the phase difference of the two neighboring harmonics and the time delay in atomic photoionization process. The atomic time delay can be presented as the sum of time delays in the xuv photon absorption and subsequent ir photon absorption (continuum-continuum or CC transition).

$$
\tau_{A}=\tau_{W}+\tau_{C C}
$$

The $\tau_{W}$ term represents the Eisenbud-Wigner-Smith time delay (or Wigner time delay or photoelectron group delay; all these terms are used interchangeably in the present context), which is defined as the energy derivative of the complex phase of the quantum amplitude of xuv absorption [1,3]. More details on the Wigner time-delay theory can be found in the review article [4]. The $\tau_{C C}$ term is modeled using the lowest order perturbation theory and asymptotic forms of the continuum wave functions, thus allowing us to obtain the former from an experimental

\footnotetext{
*a.kheifets@anu.edu.au
}

measurement [5]. By reconstructing the oscillations of SB 22 to 26 of the titanium:sapphire laser at $800 \mathrm{~nm}$, Klünder et al. [2] reported the relative time delay between the photoelectron emission from the $3 s$ and $3 p$ subshells of Ar in the photon energy range of 34 to $40 \mathrm{eV}$. Whether the $3 p$ electron was delayed relative to the $3 s$ one or vice versa was found to depend on the photon energy. This measurement was repeated later by Guénot et al. [6] and the sign of the relative time delay was reverted with the $3 s$ photoelectron being delayed relative to the $3 p$ one near the top end of the photon energy scale.

This repeated measurement was prompted by observation that the photon energy of $40 \mathrm{eV}$ fell very close to the Cooper minimum of the $3 s$ shell. The photoionization process in this region is driven very strongly by the many-electron correlation between the $3 s$ and $3 p$ subshells [7]. Such a process cannot be theoretically described using an independent electron model like the Hartree-Fock (HF) theory. So the interpretation of the two-photon interferometric measurement [2] based on this theory had to be re-evaluated. A more adequate model that accounts for intershell correlation in noble gas atoms is the random-phase approximation with exchange (RPAE or RPA; both acronyms are used here interchangeably) [8]. However, even after including the RPA corrections, the agreement between theory and experiment did not improve [6].

Theoretical interpretation of the attosecond streaking measurement of Schultze et al. [1] is also not straightforward. The group delay difference between the $2 p$ and $2 s$ subshells in $\mathrm{Ne}$ calculated in the HF approximation is only 6.2 as [3]. With the added RPA correction of 2.2 as, it accounts for less that a half of the experimental value of $21 \pm 5$ as. More accurate simulations that accounted for both the xuv and ir fields returned somewhat larger values of $10.2 \pm 1.3$ as [9] and $\sim 12$ as [10]. These values are still far too small to match the experimental result.

Even though the streaking ir field is relatively weak, its interplay with the long-range Coulomb potential of the ionic core (the so-called Coulomb-laser coupling, CLC) makes an additional contribution to the streaking time delay [11-14]. Similar to Eq. (1), the streaking time delay can be written as

$$
\tau_{s}=\tau_{W}+\tau_{\mathrm{CLC}}
$$

It was suggested in Ref. [11] that $\tau_{\text {CLC }}$ should also include the effect of the short-range part of the core potential and hence Eq. (2) should be modified to contain twice the Wigner time 
delay. This would have resolved the difference between the theoretical [1,3] and experimental [1] time delays. However, subsequent investigation on the model two-electron system, which mimicked the energy levels of the valence shell of $\mathrm{Ne}$, proved that this conjecture is invalid and that Eq. (2) holds. Therefore, the controversy surrounding the experiment [1] still remains unresolved. It should be finally mentioned that although the two corrections $\tau_{\text {CLC }}$ and $\tau_{C C}$ were introduced independently and attributed seemingly to different effects, they are in fact identical and originate from the same phase shift of the two-photon, above-threshold matrix element, c.f. Ref. [5].

In the present paper, we concentrate on the Wigner component $\tau_{W}$ which enters the atomic time delay (1) and the streaking time delay (2) measured in the attosecond interferometric and streaking experiments, respectively. The corresponding corrections to the Wigner time delay, $\tau_{C C}$ and $\tau_{\mathrm{CLC}}$, are more or less universal and can be readily evaluated $[10,14]$. For two-electron atomic transitions, like photoionization with excitation and double photoionization, which are strongly driven by electron correlation, the streaking time delay (2) is further modified by the CLC effect on the interelectron interaction [15]. These two-electron processes, however, are outside the scope of the present study. The target polarization by the streaking ir field can also be safely ignored as it should be minimal for tightly bound closed-shell atoms.

In the present work, we perform systematic investigation of the Wigner time delay in a series of noble-gas atoms from $\mathrm{Ne}$ to $\mathrm{Xe}$ across a wide range of photon energies. We demonstrate that in heavier noble gases, beyond $\mathrm{Ne}$, the intershell correlation, in the form of direct Coulomb interaction between atomic electrons assigned to different valence sub-shells, has a strong effect on the photoionization process in general and the Wigner time delay in particular. To account for this direct interelectron interaction, we employ the RPA method [16]. This method can be viewed as an extension of the HF theory. The latter accounts for the Coulomb interelectron interaction only indirectly by including some part of it in the self-consistent one-electron potential. On the contrary, the RPA method accounts for a significant part of the direct interelectron interaction that results in creation of pairwise electron-hole excitations. When more complex excitations of two-electron-two-hole states are important (see, e.g., Ref. [17]), alternative methods like the $R$ matrix [18] can provide more accurate results.

We validate our computational technique by making an extensive comparison between the calculated and experimental valence-shell photoionization cross sections. Based on this validation, we make specific predictions for the Wigner time delay and perform further comparison with available experimental time-delay data. More generally, we demonstrate that the Wigner time delay contains important phase information that enables attosecond time-delay measurements to reveal various fundamental aspects of atomic physics.

The paper is organized as follows. In Sec. II we introduce our computational models for the independent electron descriptions and that with account for the intershell correlations. In Sec. III we present our numerical results for outer valence $n s$ and $n p$ subshells in $\mathrm{Ne}$ and $\mathrm{Ar}$ and $n s, n p,(n-1) d$ subshells in $\mathrm{Kr}$ and Xe. We conclude in Sec. IV by revealing the systematic trends in time delay of noble gases driven by the peculiarities of the elastic scattering phases and many-electron correlations.

\section{THEORETICAL MODEL}

\section{Independent-electron HF model}

We adopt the photoionization formalism as outlined in the monograph [16]. We evaluate the one-photon dipole matrix element $\left\langle\psi_{\boldsymbol{k}}^{(-)}|\hat{z}| \phi_{i}\right\rangle$ of the transition from a bound state $i$ to an incoming continuous wave with the given photoelectron momentum $\boldsymbol{k}$. The magnitude of the momentum is restricted by the energy conservation $E \equiv k^{2} / 2=\omega+\varepsilon_{i}$, where $\omega$ is the photon energy. The atomic units are used throughout the paper with $e=m=\hbar=1$ and the atomic unit of time is approximately equal to 24.2 as.

We split the radial and angular dependence in the initial state $\phi_{i}(\boldsymbol{r})=Y_{l_{i} m_{i}}(\hat{\boldsymbol{r}}) R_{n_{i} l_{i}}(r)$ and use the partial wave expansion in the final state

$$
\psi_{\boldsymbol{k}}^{(-)}(\boldsymbol{r})=\frac{(2 \pi)^{3 / 2}}{k^{1 / 2}} \sum_{l m} i^{l} e^{-i \delta_{l}(E)} Y_{l m}^{*}(\hat{\boldsymbol{k}}) Y_{l m}(\hat{\boldsymbol{r}}) R_{E l}(r),
$$

where the radial orbitals are normalized to energy $\left\langle E l \| E^{\prime} l\right\rangle=$ $\delta\left(E-E^{\prime}\right)$ and have the asymptotics at infinity

$$
\left.P_{E l}(r)\right|_{r \rightarrow \infty}=\sqrt{\frac{2}{\pi k}} \frac{1}{r} \sin \left(k r-\frac{l \pi}{2}+\delta_{l}\right) .
$$

We align the quantization axis $z$ with the polarization axis of light and write the dipole operator in the length gauge as $\hat{z}=\sqrt{4 \pi / 3} r Y_{10}(\hat{\boldsymbol{r}})$. We perform the spherical integration to arrive at the following expression:

$$
\begin{aligned}
\left\langle\psi_{\boldsymbol{k}}^{(-)}|\hat{z}| \phi_{i}\right\rangle= & \frac{(2 \pi)^{3 / 2}}{k^{1 / 2}} \sum_{\substack{l=l_{i} \pm 1 \\
m=m_{i}}} e^{i \delta_{l}(E)} i^{-l} Y_{l m}(\hat{\boldsymbol{k}}) \\
& \times\left(\begin{array}{ccc}
l & 1 & l_{i} \\
m & 0 & m_{i}
\end{array}\right)\left\langle E l\|r\| n_{i} l_{i}\right\rangle .
\end{aligned}
$$

Here the reduced dipole matrix element, stripped of all the angular momentum projections, is defined as

$$
\left\langle E l\|r\| n_{i} l_{i}\right\rangle=\hat{l}_{i}\left(\begin{array}{ccc}
l & 1 & l_{i} \\
0 & 0 & 0
\end{array}\right) \int r^{2} d r R_{E l}(r) r R_{n_{i} l_{i}}(r),
$$

where $\hat{l}=\sqrt{2 l+1}$. The partial photoionization cross section for the transition from an occupied state $n_{i} l_{i}$ to the photoelectron continuum state $E l$ is calculated as

$$
\sigma_{n_{i} l_{i} \rightarrow E l}(\omega)=\frac{4}{3} \pi^{2} \alpha a_{0}^{2} \omega\left|\left\langle E l\|r\| n_{i} l_{i}\right\rangle\right|^{2} .
$$

Here $\alpha$ is the fine structure constant and $a_{0}$ is the Bohr radius.

The basis of occupied atomic states $\left.\| n_{i} l_{i}\right\rangle$ is defined by the self-consistent HF method and calculated using computer code [19]. The continuum electron orbitals $\langle E l \|$ are defined within the frozen-core HF approximation and evaluated using the computer code given in Ref. [20]. These states are found in the combined field of the nucleus and the HF potential of the frozen electron core. So the photoelectron scattering phase $\delta_{l}(E)$ delivered by this method contains both the long-range Coulomb and the short-range Hartree-Fock components.

We note that the reduced matrix element (5) is real and thus the complex phase of the dipole matrix element (4) is 
defined by the scattering phases $\delta_{l_{i} \pm 1}(E)$. According to Fano's propensity rule [21], the dipole transition with the increased momentum $l=l_{i}+1$ is usually dominant. In such a situation, the photoemission group delay is approximately given by $\tau_{W}=d \delta_{l} / d E$.

\section{Intershell correlation}

To include intershell correlation effects, we employ the RPA model [16]. In this approximation, the reduced dipole matrix element (5) is replaced by its correlated counterpart $\left\langle E l\|D\| n_{i} l_{i}\right\rangle$, which accounts for correlation between different valence subshells. This correlated matrix element is found as a solution of the system of the integral equations:

$$
\begin{aligned}
\left\langle E l\|D\| n_{i} l_{i}\right\rangle= & \left\langle E l\|r\| n_{i} l_{i}\right\rangle+\frac{1}{3} \lim _{\varepsilon \rightarrow 0^{+}} \sum_{\substack{n^{\prime} l^{\prime} \\
n_{j} l_{j}}} d E^{\prime} \\
& \times\left[\frac{\left\langle E^{\prime} l^{\prime}\|D\| n_{j} l_{j}\right\rangle\left\langle n_{j} l_{j} E l\|V\| E^{\prime} l^{\prime} n_{i} l_{i}\right\rangle}{\omega-E^{\prime}+\epsilon_{n_{j} l_{j}}+i \varepsilon}\right. \\
& \left.+\frac{\left\langle n_{j} l_{j}\|D\| E^{\prime} l^{\prime}\right\rangle\left\langle p l^{\prime} E l\|V\| n_{j} l_{j} n_{i} l_{i}\right\rangle}{\omega+E^{\prime}-\epsilon_{n_{j} l_{j}}}\right] .
\end{aligned}
$$

Here the combined sum plus integral sign incorporates both the summation over the discrete excited states $n^{\prime} l^{\prime}$ with the energy $\epsilon_{n^{\prime} l^{\prime}}$ and the integration over the continuum $d E^{\prime}$ from the threshold to infinity. The Coulomb matrix contains both the direct and the exchange parts $V=2 U-W$. That explains the term exchange in the name $\operatorname{RPA}(\mathrm{E})$. The direct Coulomb matrix is expressed as

$$
\begin{aligned}
\left\langle n_{j} l_{j} E l\|U\| E^{\prime} l^{\prime} n_{i} l_{i}=\right. & \hat{l}^{\prime} \hat{l}_{i} \hat{l}_{j}\left(\begin{array}{ccc}
l & 1 & l_{i} \\
0 & 0 & 0
\end{array}\right)\left(\begin{array}{ccc}
l^{\prime} & 1 & l_{j} \\
0 & 0 & 0
\end{array}\right) \\
& \times R_{l, l^{\prime}, l_{i}, l_{j}}^{(1)}\left(E, E^{\prime}, n_{i}, n_{j}\right),
\end{aligned}
$$

where $R^{(1)}$ is a Slater integral [16]. In the exchange matrix, the electron $E l$ and the hole $n_{j} l_{j}$ states are swapped.

The RPA equations are represented graphically in Fig. 1. Here the straight line with an arrow to the right or left represents electron (continuum) or hole (bound) states, respectively. The wavy line exhibits the Coulomb interaction. The dashed line is used to display a photon of the frequency $\omega$. The shaded circle is used to represent the correlated dipole matrix element, whereas the bare matrix element is exhibited by a three-pronged vertex. The Coulomb interaction matrices $\left\langle n_{j} l_{j} E l\|V\| E^{\prime} l^{\prime} n_{i} l_{i}\right\rangle$ and $\left\langle E^{\prime} l^{\prime} E l\|V\| n_{i} l_{i} n_{j} l_{j}\right\rangle$ describe the so-called time-forward and time-reverse correlation processes
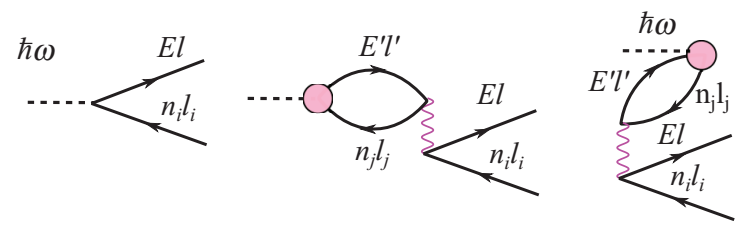

FIG. 1. (Color online) Graphical representation of the RPA equations (7). Left: noncorrelated dipole matrix element. Center: time-forward process. Right: time-reverse process. which are exhibited by the second and third diagrams (from left to right). In the time-forward process, the photon absorption is followed by the interelectron interaction in the form of creation of the virtual electron-hole pair in the neighboring subshell. In the time-reverse process, the virtual electron-hole pair is created before the photon absorption takes place. Because the time-forward process is real in a sense that it conserves the energy of the system while the time-backward process is virtual, the time-forward process makes a stronger contribution to the photoionization process. However, for the completeness and gauge invariance of the theory, both processes should be taken into account.

We solve the system of integral equations (7) using a slightly modified version of the computer code given in Ref. [22]. The energy integration in the time-forward term of Eq. (7) (second line) contains a pole and the RPA matrix element acquires an imaginary part and therefore an extra phase $\arg \left\langle E l\|D\| n_{i} l_{i}\right\rangle$. However, this phase does not enter the partial photoionization cross section $n_{i} l_{i} \rightarrow E l$, which is obtained from the squared matrix element. To get access to the phase information, one has to evaluate the angular asymmetry parameter $\beta$ which contains the phase difference between the two photoionization channels $l=l_{i} \pm 1$ when $l_{i} \neq 0$ [16]. The photoelectron group delay, which is the energy derivative of the phase of the complex photoionization amplitude, gives an alternative access to the phase information. It is evaluated as

$$
\tau=\frac{d}{d E} \arg f(E) \equiv \operatorname{Im}\left[f^{\prime}(E) / f(E)\right] .
$$

Here the photoionization amplitude $f(E)$ is given the partial wave expansion

$$
\begin{aligned}
f(E) \propto & \sum_{l=l_{i} \pm 1} e^{i \delta_{l}} i^{-l} Y_{l m}(\hat{\boldsymbol{k}})(-1)^{m}\left(\begin{array}{ccc}
l & 1 & l_{i} \\
-m & 0 & m_{i}
\end{array}\right) \\
& \times\left\langle E l\|D\| n_{i} l_{i}\right\rangle .
\end{aligned}
$$

The amplitude $f(E)$ is evaluated in the forward direction $\boldsymbol{k} \| \hat{z}$, which is usually the case in the attosecond-time-delay measurements. In this case, $Y_{l m}(\hat{\boldsymbol{k}} \| \hat{z})=\hat{l}(4 \pi)^{-1 / 2} \delta_{m 0}$ and hence $m_{i}=0$ also. It has to be noted that the phase of the amplitude (10) contains the contribution of the HF phases $\delta_{l}$ in both photoionization channels $l=l_{i} \pm 1$ as well as the RPA correction due to the imaginary part of the RPA dipole matrix element $\left\langle E l\|D\| n_{i} l_{i}\right\rangle$. Thus the associated group delay is labeled HF + RPA and the numerical results are presented in the following section.

\section{NUMERICAL RESULTS}

\section{A. Neon $2 s$ and $2 p$ subshells}

On the top panel of Fig. 2 we present the partial photoionization cross sections of valence-shell photoionization of Ne. The HF cross sections are shown by the dashed (blue online) lines and the RPA cross sections are given by the solid (red online) line. The recommended experimental data by Bizau and Wuilleumier [23] are displayed with error bars. In the RPA calculation, we substitute the HF bound-state energies with the the experimental ionization thresholds $\epsilon_{2 p_{3 / 2}}=21.56 \mathrm{eV}$ and $\epsilon_{2 s}=48.47 \mathrm{eV}$ [24], which are indicated on the upper boundary of the panel. We see that the account for the 

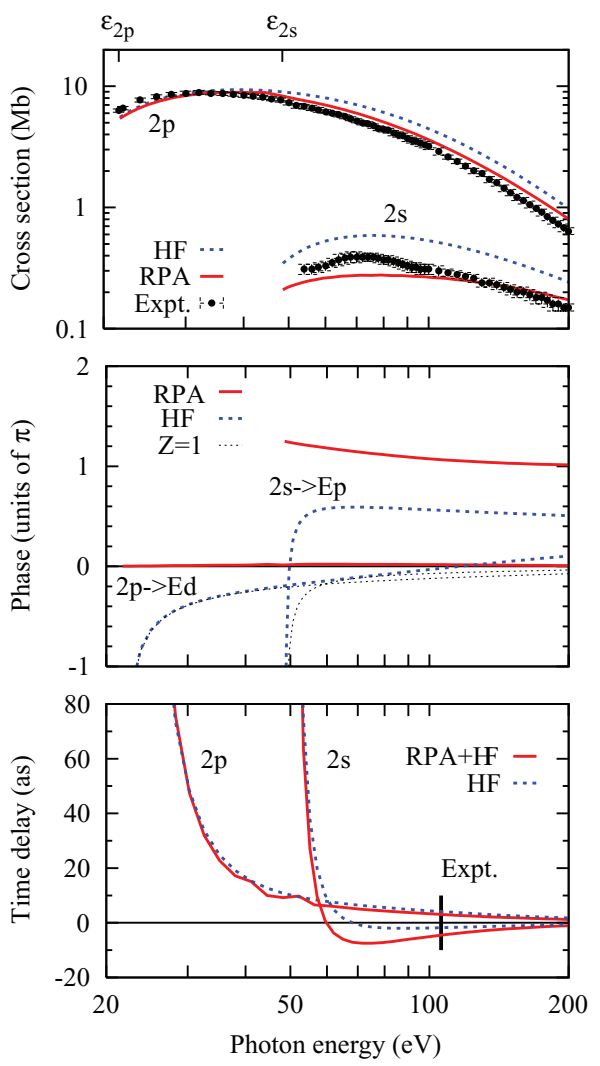

FIG. 2. (Color online) Top: the partial photoionization cross sections of the $2 s$ and $2 p$ subshells of Ne. The HF and RPA calculations are shown by the dashed (blue online) and solid (red online) lines, respectively. The recommended experimental data by Bizau and Wuilleumier [23] are displayed with error bars. Middle: elastic scattering phases in the field of the $\mathrm{Ne}^{+}$ion for the $2 s \rightarrow E p$ and the dominant $2 p \rightarrow E d$ channels (dotted [blue online] line) and the RPA phases (solid [red online] line). The thin dotted line visualizes the Coulomb phase with $Z=1$. Bottom: the phase derivatives are converted to the units of the group delay. The vertical bar at the photon energy of $106 \mathrm{eV}$ visualizes the relative time delay between the $2 p$ and $2 s$ subshells of 21 as as measured by Schültze et al. [1].

RPA correlation between the $2 s$ and $2 p$ subshells improves the calculated cross sections and makes then closer to the experimental data.

We note that even though agreement between theory and experiment is improved in the RPA model, there is a visible difference between the calculated and measured cross sections, especially for the $2 s$ subshell. This difference may arise from the fact that not all the many-electron correlations are accounted for by the RPA model, which includes pairwise electron-hole virtual excitations. Other processes like admixture of the two-hole-one-electron states to the pure one-hole state in the singly charged ion are not included in the RPA model. This admixture is responsible for the shift in atomic ionization potentials relative to the corresponding HF binding energies as well as appearance of the satellite lines in the photoionization spectra [25]. These effects cannot be accounted for $a b$ initio in the RPA model. Phenomenologically, they are partly compensated by using the experimental ionization potentials instead of the HF energies $\varepsilon_{i}$ in the RPA equations (7).
On the middle panel, we show the elastic scattering phases in the field of the $\mathrm{Ne}^{+}$ion for the $2 s \rightarrow E p$ and the dominant $2 p \rightarrow E d$ channels. The HF phases $\delta_{p}(E)$ and $\delta_{d}(E)$ are plotted with the dashed (blue online) line. The RPA phases $\arg \langle k p\|D\| 2 s\rangle$ and $\arg \langle k d\|D\| 2 p\rangle$ are displayed with the solid (red online) line. The thin dotted line visualizes the Coulomb phase $\sigma_{l}(E)=\arg \Gamma\left(1+l-i Z_{\text {eff }} / \sqrt{2 E}\right)$ with the effective charge $Z_{\text {eff }}=1$. This phase shows the contribution of the long-range Coulomb potential to the HF phase $\delta_{l}(E)$, which is strongly dominant at small kinetic energies of the photoelectron. The phase shift due to the short-range potential, i.e., the difference of the total phase and the Coulomb phase, is related to the quantum defect according to the Levinson-Seaton theorem $\delta_{l}(k \rightarrow 0)-\sigma_{l}(k \rightarrow 0)=$ $\mu_{l}(\infty) \pi$ [26]. For a neutral target, the scattering phase at zero energy is related to the number of the bound target states $N_{l}$ by the Levinson's theorem $\delta_{l}(k \rightarrow 0)=N_{l} \pi$. In the absence of the Coulomb potential, the $2 s \rightarrow E p$ phase would tend to one unit of $\pi$ at $k \rightarrow 0$ as there is one occupied $n p$ subshell in the $\mathrm{Ne}^{+}$ion with $n=2$. With the Coulomb potential taken into account, $\delta_{2 s \rightarrow k p}(k \rightarrow 0)-$ $\sigma_{2 s \rightarrow k p}(k \rightarrow 0)=0.88 \pi$, where $\mu_{l=1}=0.88$ is the quantum defect calculated from fitting the $n p$ orbital energies in the $\mathrm{Ne}^{+}$ion $\epsilon_{n p} \propto-\left(n-\mu_{l=1}\right)^{2}$ for $n>2$. As the Coulomb phase tends to zero rapidly away from the threshold, the HF phase stays rather flat at the value determined by the corresponding quantum defect. We may associate this behavior with the Levinson theorem, even though this theorem is strictly valid only at $k \rightarrow 0$. Similarly, the $2 p \rightarrow E d$ phase would tend to zero as there are no occupied $d$ shells left behind. The Coulomb logarithmic singularity changes this behavior radically and sends the scattering phases to large negative values near the threshold. It has to be noted that when the Coulomb behavior of the phases and associated group delays becomes dominant at low photoelectron energies, the measurement-induced components in the experiments, i.e., the Coulomb-laser coupling in attosecond streaking $[12,13]$ or the continuum-continuum contribution in interferometric two-photon measurements [2,5,6], will be large and the group delay as presented in this paper can only be accessed if those corrections are properly accounted for.

The RPA phase in the $2 p \rightarrow E d$ channel is hardly distinguishable from zero. This observation is consistent with a very small change that the RPA correction causes to the partial photoionization cross section shown on the top panel. The RPA phase in the $2 s \rightarrow E p$ channel is large but rather flat and changes slowly with the photon energy. This is consistent with the $2 s$ partial photoionization cross section, which is affected by the intershell correlation with $2 p$ across the whole range of the studied photon energies.

The bottom panel of Fig. 2 displays the photoelectron group delay calculated as the energy derivative of the phase of the photoionization matrix element. The HF group delay in the dominant photoionization channel is calculated as $\tau_{W}^{\mathrm{HF}}$ (as) $=$ $k^{-1} d \delta_{l} / d k \times 24.2$. Here $E=k^{2} / 2$ is the photoelectron energy in atomic units and one unit of time is equal approximately to 24.2 as. In the existing code, the continuous electron orbitals are calculated on the regular momentum grid and numerical differentiation over the momentum, rather than energy, is easier to implement. The fine grid of 0.05 a.u. of photoelectron 
momentum is sufficient for an accurate numerical differentiation. Similarly, the combined RPA + HF time delay is calculated as $\tau_{W}^{\mathrm{RPA}+\mathrm{HF}}($ as $)=\operatorname{Im}\left[k^{-1} f^{\prime}(k) / f(k)\right] \times 24.2$. Here the photoionization amplitude (10) is evaluated in the $z$-axis direction. We see that the HF time delay in the dominant $2 p \rightarrow E d$ channel accounts for almost the whole time delay in photoemission from the $2 p$ subshell. There is some oscillation visible due to the autoionizing resonances near the $2 s$ threshold which is absent in the HF approximation. Overall, the $2 p$ time delay is always positive and rapidly decreasing function of the photon energy. This is explained by the monotonously decreasing HF phase in the $d$-partial wave, which is driven by the Coulomb logarithmic singularity. The situation is different in the $2 s \rightarrow E p$ channel. Here the HF phase crosses over from the Coulomb behavior at low photoelectron kinetic energy to the Levinson behavior at larger energies. In result, the phase derivative and, consequently, the time delay change their sign from positive and negative towards the larger photon energies. The RPA correction to the time delay is always negative. Hence the photoemission from the $2 s$ subshell seems to be ahead of that of the $2 p$ subshell at around $100-\mathrm{eV}$ photon energy mark where the measurement of Schültze et al. [1] was taken (shown as a vertical bar in the figure). According to Eq. (2), to make a comparison of the present calculation with the experiment, we have to add to the Wigner time-delay difference between the $2 p$ and $2 s$ subshells $\Delta \tau_{W}=8.4$ as with the difference between the corresponding CLC corrections $\Delta \tau_{\mathrm{CLC}}=3.5$ as [14]. The resulting time delay difference $\Delta \tau_{s}=11.9$ as, which is very similar to that reported in Ref. [10] but only half of the experimental value of $21 \pm 5$ as.

\section{Argon $3 s$ and $3 p$ subshells}

An analogous set of data for Ar $3 s$ and $3 p$ subshells is shown in Fig. 3. On the top panel we make a comparison of the HF (dashed [blue online] line) and the RPA (solid [red online] line) partial photoionization cross sections with the experimental data by Möbus et al. [27] for $3 s$ subshell and Samson and Stolte [28] for the sum of $3 s$ and $3 p$ subshells. The experimental ionization thresholds $\epsilon_{3 \mathrm{p}_{3 / 2}}=15.76 \mathrm{eV}$ and $\epsilon_{3 s}=29.24 \mathrm{eV}$ [24] are indicated on the upper boundary of the panel. These partial photoionization cross sections are qualitatively different from those of Ne shown in Fig. 2. First, the $3 p$ cross section in Ar displays the Cooper minimum, whereas the nodeless $2 p$ orbital does not [29]. Second, the intershell correlation changes completely the $3 s$ cross section, which also displays a deep Cooper-like minimum at a slightly smaller photon energy. The RPA calculation reproduces these features in fair agreement with the experiment.

The HF phases in Ar behave similarly to the analogous case of $\mathrm{Ne}$ except that the $3 s \rightarrow E p$ phase would tend to $2 \pi$ in the absence of the Coulomb singularity as there are two occupied $n p$ shells in the $\mathrm{Ar}^{+}$ion. With the Coulomb potential taken into account, $\delta_{3 s \rightarrow k p}(k \rightarrow 0)-\sigma_{3 s \rightarrow k p}(k \rightarrow$ $0)=1.73 \pi$, where the corresponding value of the quantum defect in $\mathrm{Ar}^{+}$is $\mu_{l=1}=1.73$. The RPA phases in Ar are very different from $\mathrm{Ne}$. When the cross section goes through the Cooper minimum, the corresponding phase makes a jump of $\pi$ in the $3 s \rightarrow E p$ amplitude and $-\pi$ in the $3 p \rightarrow E d$ amplitude. This jump is easy to understand. If the amplitude

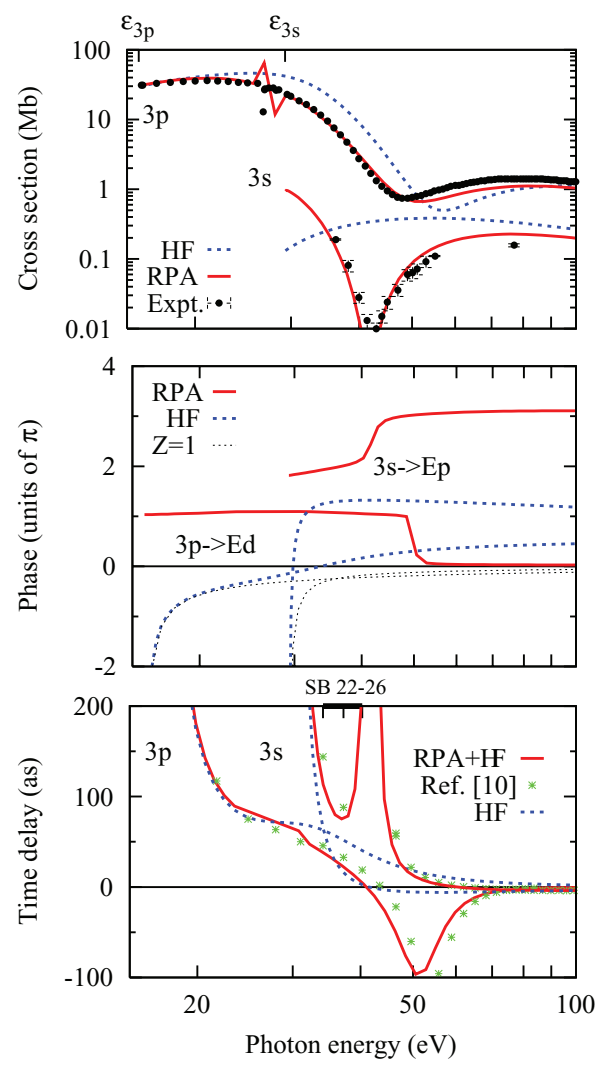

FIG. 3. (Color online) Top: the partial photoionization cross sections of the $3 s$ and $3 p$ subshells of Ar. The HF and RPA calculations are shown by the dashed (blue online) and solid (red online) lines, respectively. The experimental data for $3 s$ [27] and for $3 s+3 p$ [28] are displayed with error bars. Middle: elastic scattering phases in the field of the $\mathrm{Ar}^{+}$ion for the $3 s \rightarrow E p$ and the dominant $3 p \rightarrow E d$ channels (dotted [blue online] line) and the RPA phases (solid [red online] line). Bottom: the phase derivatives are converted to the units of the group delay. The asterisks (green online) display the calculation [10] corrected for experimental ionization thresholds.

was real and had a node, it would simply change its sign, which would amount to adding a phase factor of $\pi$ in the complex number representation. Incidentally, this jump was investigated in an earlier model calculation [30], which established the validity of the attosecond streaking technique for the phase measurements.

This jump of $\pi$ has a dramatic effect on the time delay, which is shown on the bottom panel of Fig. 3. It drives the time delay in the $3 s$ subshell to very larger numbers in several hundreds of attoseconds. The situation is less dramatic for the $3 p$ subshell. Here the normally weak transition $3 p \rightarrow E s$ takes over near the Cooper minimum of the strong $3 p \rightarrow E d$ transition and the resulting time delay does not go below -100 as. We note that there is a strong variation of phase near the autoionization resonances in the $3 p$ photoionization, which is seen on the top panel of Fig. 3. We do not show this variation in the phase and time-delay plots for clarity of presentation. Anyway, these resonances are far too narrow to be detected in time-delay measurements at the present energy resolution.

One can compare the significant time delay near the Cooper minimum with the delay time in Breit-Wigner resonant 
TABLE I. Relative time delay between the photoemission from the $3 s$ and $3 p$ subshells in $\mathrm{Ar}$ at three fixed photon energies corresponding to the SB 22 to 26 in the experiment of Guénot et al. [6]. The experimental uncertainty is \pm 50 as. The MCHF calculation [33] has typical error bars of \pm 40 as due to resonance structure.

\begin{tabular}{lcrrrr}
\hline \hline & & \multicolumn{4}{c}{$\tau_{W}^{3 s}-\tau_{W}^{3 p}$ (as) } \\
\cline { 3 - 6 } SB & $\omega(\mathrm{eV})$ & HF & RPA & MCHF & Expt. \\
\hline 22 & 34.1 & 3 & 76 & 45 & 70 \\
24 & 37.2 & -36 & 53 & 10 & -30 \\
26 & 40.3 & -38 & 215 & -5 & 50 \\
\hline \hline
\end{tabular}

scattering $t_{d}=2 / \Gamma$ with $\Gamma$ being the resonant width at half maximum of the cross section [31]. In the case of the Cooper minimum in Ar, which is roughly 0.5 a.u. of energy wide, the time delay is expected to be 4 atomic units of time, which equates to about 100 as. Of course, this is a very rough estimate and the actual time delay is not constant but varies across the Cooper minimum. The steepness of this variation can only be estimated from an accurate numerical calculation.

On the upper boundary of the bottom panel, we indicate the photon energies corresponding to the SB 22 to 26 of the titanium:sapphire laser at $800 \mathrm{~nm}$ used in the two-photon interferometric experiments [2,6]. We see that at this photon energy range, the RPA correction changes completely the sign of the relative $3 p / 3 s$ time delay. In the HF approximation, the $3 p$ photoemission is delayed more that the $3 s$ ones. The intershell correlation changes this ordering completely. With the RPA correction, it is the $3 s$ that is delayed more than the $3 p$. This is an important, strong and qualitative result which is related to the Cooper minima in the corresponding partial photoionization cross sections. This result is confirmed by an alternative time-independent calculation by Dahlström et al. [10] with a similar account for many-electron correlations as in RPA. As compared to the original calculation presented in Ref. [10], the group-delay data shown on the bottom panel of Fig. 3 are corrected for the experimental ionization potentials [32]. Without this correction, the HF ionization potential of the $3 s$ subshell $\varepsilon_{3 s}=34.7 \mathrm{eV}$ makes the SB 22 inaccessible.

A strong modification of the relative time delay between the $3 p$ and $3 s$ subshells in Ar is more clearly seen in Table I, where we present the time-delay difference $\tau_{3 s}-\tau_{3 p}$ in the HF and RPA approximations and compare it with the experimental data of Ref. [6]. Even a fairly large uncertainty of \pm 50 as cannot reconcile the experimental data with either of the calculations. In the same table, we present results of a multiconfigurational Hartree-Fock (MCHF) close-coupling calculation [33]. In this calculation, the Cooper minimum was displaced to significantly larger photon energies, which were not probed experimentally. Hence, the time-delay difference at the SB 26 was not affected by this minimum as strongly as in the present RPA calculation.

\section{Krypton $4 p, 4 s$, and $3 d$ subshells}

Our results for the $4 p, 4 s$, and $3 d$ photoionization of $\mathrm{Kr}$ are displayed in Fig. 4. In the top panel we make a comparison of the HF (dashed [blue online] line) and the RPA (solid [red
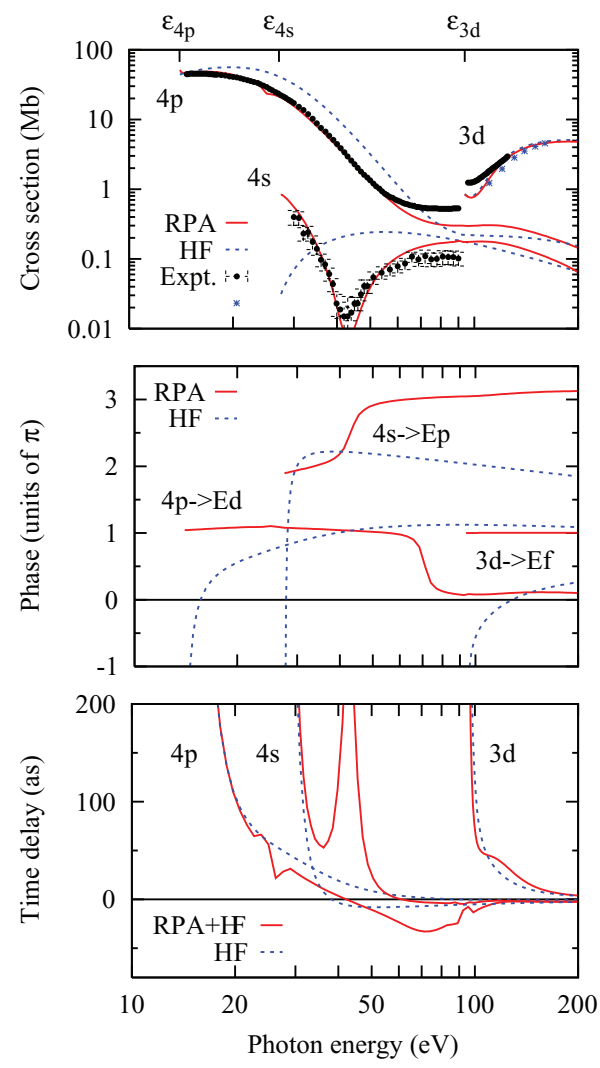

FIG. 4. (Color online) Top: the partial photoionization cross sections of Kr. The HF and RPA calculations are shown by the dashed (blue online) and solid (red online) lines, respectively. The experimental data from Ref. [34] for $4 s$ and Ref. [28] for $4 p+3 d$ are displayed with error bars. The data from Ref. [35] for $3 d$ are displayed with asterisks. Middle: elastic scattering phases in the field of the $\mathrm{Kr}^{+}$ion for the $4 s \rightarrow E p$ and the dominant $4 p \rightarrow E d$ channels (dotted [blue online] line) and the RPA phases (solid [red online] line). Bottom: the phase derivatives are converted to the units of the group delay.

online] line) partial photoionization cross sections with the experimental data from Ref. [34] for $4 s$ and Ref. [28] for $4 p+$ $3 d$ (error bars). The data from Ref. [35] for $3 d$ are displayed with asterisks. The experimental ionization thresholds $\epsilon_{4 \mathrm{p}_{3 / 2}}=$ $14.00 \mathrm{eV}, \epsilon_{4 s}=27.51 \mathrm{eV}$ [24], and $\epsilon_{3 d_{5 / 2}}=93.83 \mathrm{eV}$ [36] are indicated on the upper boundary of the panel. The $4 p$ and $4 s$ cross sections in $\mathrm{Kr}$ behave similarly to the $3 p$ and $3 s$ cross sections in Ar (see the top panel of Fig. 3). The $4 p \rightarrow E d$ cross section goes through its Cooper minimum, which is offset somewhat by the weaker $4 p \rightarrow E s$ channel. So the total $4 p$ cross section displays a shoulder rather than a true minimum. The $4 s$ cross section is driven strongly by its intershell correlation with $4 p$ to a very deep minimum, which is missed completely in the HF approximation. The $3 d$ cross section from its threshold displays a strong maximum associated with its shape resonance. This resonance is known to be due to electron correlation within a single shell [37] and indeed the $3 d$ photoionization cross section is well described by the HF approximation.

The HF phases in $\mathrm{Kr}$ (middle panel of Fig. 4) behave similarly to the analogous cases of $\mathrm{Ne}$ and Ar except that the $4 s \rightarrow E p$ phase would tend to $3 \pi$ and the $4 p \rightarrow E d$ phase 
would tend to $\pi$ in the absence of the Coulomb potential. With this potential, the HF phases are determined by the corresponding quantum defect values $\mu_{l=1}=2.67$ and $\mu_{l=2}=$ 1.04. The RPA phases in $\mathrm{Kr}$ are also similar to Ar. Every time the cross section goes through the Cooper minimum, the corresponding phase makes a jump of $\pi$ : upward in the $4 s \rightarrow$ $E p$ amplitude and downward in the $4 p \rightarrow E d$ amplitude. The RPA phase in the $3 d \rightarrow E f$ transition is rather stationary.

This behavior of the phases translates into the corresponding time delays plotted on the bottom panel of Fig. 4. The RPA time delay in $4 p$ subshell is not dramatically different from the HF calculation. Even though the dominant $4 p \rightarrow E d$ transition displays a Cooper minimum, it is offset by the weak $4 p \rightarrow E s$ transition and is not as prominent in the total $4 p$ cross section as in the $3 p$ cross section of Ar. There are some variation of the time delay near the autoionizing resonances close to the $4 s$ threshold, which are seen in the RPA calculation but not in HF one. The time delay in the $3 d$ subshell is almost entirely due to intrashell effects and the HF and RPA results are very close. The situation is very different in the $4 s$ subshell, where the time delay is strongly affected by the intershell correlation with the $4 p$ subshell and reaches 300 as in its peak. Similarly to Ar, there is a complete reversal of the relative time delay between the $4 p$ and $4 s$ subshells in the RPA calculation in comparison with the HF one.

\section{Xenon $5 p, 5 s$, and $4 d$ subshells}

The analogous set of data for the $5 p, 5 s$, and $4 d$ subshells of Xe is presented in Fig. 5. On the top panel we compare the partial photoionization cross-sections in the HF (dashed [blue online] line) and RPA (solid [red online] line) approximations with the experimental data $[38,39]$, which are shown with the asterisks (blue online) for $5 s$ and error bars for $5 p$ and $4 d$. The experimental ionization thresholds $\epsilon_{5 \mathrm{p}_{3 / 2}}=12.13 \mathrm{eV}$, $\epsilon_{2 s}=23.40 \mathrm{eV}$ [24] and $\epsilon_{4 d_{5 / 2}}=67.50 \mathrm{eV} \mathrm{[40]} \mathrm{are} \mathrm{indicated}$ on the upper boundary of the panel.

Below the $4 d$ ionization threshold, the $5 s$ and $5 p$ cross sections in Xe behave similarly to the $4 s$ and $4 p$ subshells in $\mathrm{Kr}$ (top panel of Fig. 4). However, above this threshold, the $4 d$ subshell goes through a very steep shape resonance, sometimes even called a "giant resonance." This resonance is then turns into a Cooper minimum. By strong intershell interaction, this behavior is replicated in the $5 p$ and $5 s$ partial photoionization cross sections, which are well reproduced by the RPA calculation. Accordingly, the corresponding RPA phases displays steep $\pi$ jumps (middle panel), which are reflected in the corresponding time delays (bottom panel). In the case of the $5 s$ subshell, the RPA phase jump near the Cooper minimum mergers with the Coulomb singularity and produces a very large, nearly 300 -as time delay at the photon energies below $30 \mathrm{eV}$. The $5 p$ subshell shows a large and negative time delay due to its Cooper minimum at around $50 \mathrm{eV}$. Both the $5 s$ and $5 p$ subshells display a large and negative time delay near the local cross-section minima around $150 \mathrm{eV}$ induced by the correlation with the $4 d$ subshell. The time delay in the $4 d$ subshell is driven from the strongly positive due to the Coulomb singularity at low photon energies to a large negative jump near the Cooper minimum at about $180 \mathrm{eV}$. At larger energies, the cross sections are rather structureless and there is no significant time-delay variations.
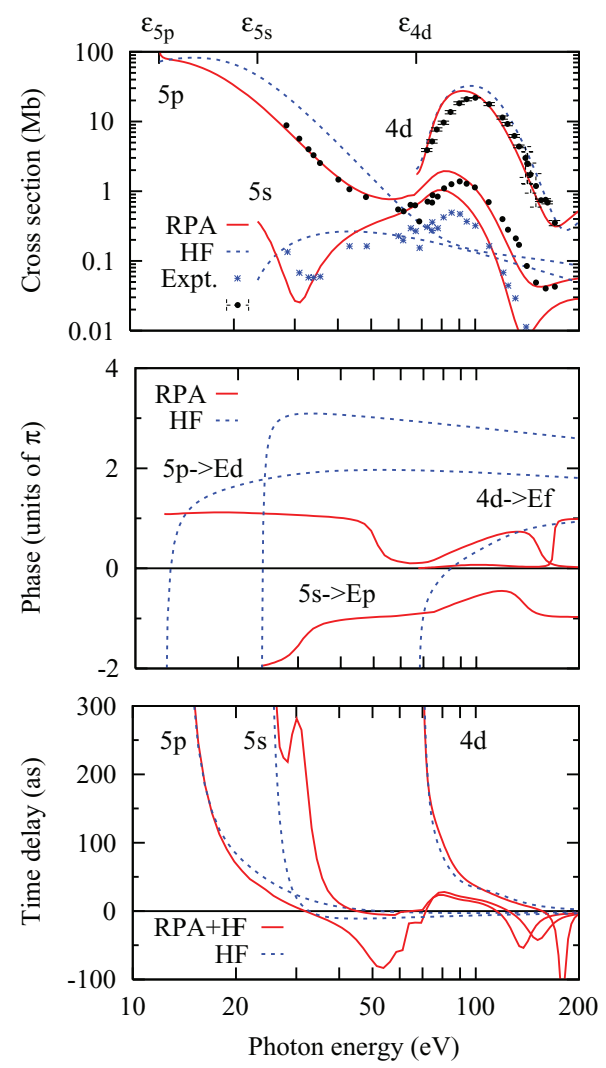

FIG. 5. (Color online) Top: the partial photoionization cross sections of Xe. The HF and RPA calculations are shown by the dashed (blue online) and solid (red online) lines, respectively. The experimental data from Becker et al. [38] and Fahlman et al. [39] are shown with asterisks for $5 s$ and error bars for $5 p$ and $4 d$. Middle: elastic scattering phases in the field of the $\mathrm{Xe}^{+}$ion for the $5 s \rightarrow E p$ and the dominant $5 p \rightarrow E d$ and $4 d \rightarrow E f$ channels (dotted [blue online] line) and the RPA phases (solid [red online] line). Bottom: the phase derivatives are converted to the units of the group delay.

A phase jump of $\pi$, smoothed by the interaction between the two channels, has already been observed both theoretically and experimentally by analyzing the anisotropy parameter in photoionization of Xe $5 p$ subshell [41]. This parameter contains the phase shift between the two photoionization channels with $l=l_{i} \pm 1$. In the case of $5 p$ photoionization, these are $5 p \rightarrow E d$ and $5 p \rightarrow E s$ transitions. Their partial photoionization cross sections and the relative phase shift are presented on the top and bottom panels of Fig. 6. On both panels, we show the present RPA and HF calculations displayed with the solid (red online) and dotted (blue online) lines, respectively. On the bottom panel, we exhibit the RPA (open circles) and HF (filled circles) phase shifts reported in Ref. [41].

On the top panel of Fig. 6 we observe a significant shift of the Cooper minimum in the $5 p \rightarrow E d$ channel towards the lower photon energies and appearance of the secondary minimum due to the correlation with the $4 d$ subshell. In the meantime, the intershell correlation does not change the $5 p \rightarrow E s$ partial photoionization cross section in such a dramatic way. Accordingly, on the bottom panel of Fig. 6, we see a strong variation of the RPA phase shift with the two 


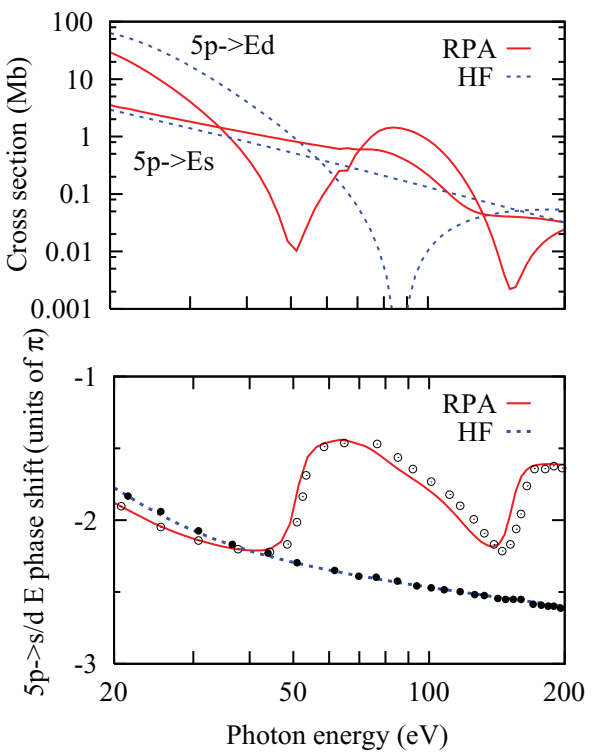

FIG. 6. (Color online) Top: Partial photoionization cross sections of $\mathrm{Xe}$ in the $5 p \rightarrow E d$ and $5 p \rightarrow E s$ channels in the RPA (solid [red online] line) and HF (dotted [blue online] line) approximations. Bottom: Phase shift between the partial $5 p \rightarrow E d$ and $5 p \rightarrow E s$ waves. The present RPA and HF calculations (solid [red online] and dotted [blue online] lines, respectively) are compared with the RPA and HF calculations reported by Zimmermann et al. [41] (open and filled circles, respectively).

successive jumps near the Cooper minima of the $5 p \rightarrow E d$ cross section. In the meantime, the $\mathrm{HF}$ calculation returns quite a smooth and monotonous phase shift. Agreement between the two sets of calculations, the present one and the one reported in Ref. [41], is rather good. A small shift between the present calculation and the reference one is most likely due to scanning and digitizing the analog data of Fig. 3 in Ref. [41].

\section{CONCLUSION}

In the present work, we perform a systematic study of the photoemission time delay from the valence shells of noblegas atoms in sequence from $\mathrm{Ne}$ to $\mathrm{Xe}$. We cover the photon energy range from the ionization threshold to $200 \mathrm{eV}$. We test the accuracy of our calculation by making comparisons with available partial photoionization cross sections. We derive the complex phase of the photoionization amplitude in the nonrelativistic HF and RPA calculations and convert it to the photoelectron group delay by taking the energy derivative.

The time-delay results display a very diverse landscape due to an interplay of three major factors. The first two are the logarithmic Coulomb singularity and the Levinson theorem which drive the photoelectron scattering phase in the field of the singly charged ion. The third factor is the phase jump of $\pi$ near the Cooper minimum, which is smoothed by the intershell interaction. The two former factors are revealed in the HF calculations, whereas the third one is most vividly reflected in the RPA calculations. Experimentally, photoionization measurements near the Cooper minima may be challenging but it is the area where the time-delay effects are expected to be largest.

These time-delay results are compared with experimental data derived from the attosecond streaking measurement [1] and the two-photon interferometric technique [6]. This comparison is inconclusive as the difference between the theoretical and experimental results clearly exceeds the reported error bars. We are fairly confident about the accuracy of the present calculation, which is tested by comparison of the partial photoionization cross sections with a large set of independent experimental data and the angular asymmetry parameters as in the case of Xe [41]. It is hard to give a numerical estimate on the accuracy of the group delay results. In lighter atoms we expect it to be within $10 \%$. For Xe, it may be more significant as suggested by larger difference between the calculated and experimental cross sections. Even for the heaviest of the atoms studied in the present work, the relativistic effects are not expected to change considerably the complex phase [41] and hence the associated group delay. It is therefore an open question why the time-delay results cannot be verified experimentally even after the corresponding CLC or $\mathrm{CC}$ corrections are made. Such a verification would be a very welcoming development both for the attosecond-time-delay measuring techniques and the complete theory of atomic photoionization. This author hopes that the present work will be a useful guide in this direction and is willing to supply the data on request to interested researchers.

\section{ACKNOWLEDGMENTS}

The author is very thankful to Marcus Dahlström for communicating his original time-delay results [10] in numerical form and for performing an additional set of calculations [32], which can be compared directly with the present work. Marcus Dahlström has also demonstrated that the instrumental corrections in the attosecond streaking and two-photon sideband interference techniqes were in fact identical. The author acknowledges Thomas Carette, who communicated the time-delay data reported in Ref. [33] in numerical form and provided a detailed explanation of the peculiarities of the MCHF method. The author thanks Vladislav Yakovlev and Anne L'Huillier for many useful and stimulating discussions and a critical reading of the manuscript. This work is supported by the Australian Research Council in the form of Discovery Grant No. DP120101805.
[1] M. Schultze et al., Science 328, 1658 (2010).

[2] K. Klünder et al., Phys. Rev. Lett. 106, 143002 (2011).

[3] A. S. Kheifets and I. A. Ivanov, Phys. Rev. Lett. 105, 233002 (2010).

[4] C. A. A. de Carvalho and H. M. Nussenzveig, Phys. Rep. 364, 83 (2002).
[5] J. Dahlström, D. Guénot, K. Klünder, M. Gisselbrecht, J. Mauritsson, A. L. Huillier, A. Maquet, and R. Taïeb, Chem. Phys. 414, 53 (2012).

[6] D. Guénot, K. Klünder, C. L. Arnold, D. Kroon, J. M. Dahlström, M. Miranda, T. Fordell, M. Gisselbrecht, P. Johnsson, J. Mauritsson et al., Phys. Rev. A 85, 053424 (2012). 
[7] J. A. R. Samson and J. L. Gardner, Phys. Rev. Lett. 33, 671 (1974).

[8] M. Y. Amusia, V. K. Ivanov, N. A. Cherepkov, and L. V. Chernysheva, Phys. Lett. A 40, 361 (1972).

[9] L. R. Moore, M. A. Lysaght, J. S. Parker, H. W. van der Hart, and K. T. Taylor, Phys. Rev. A 84, 061404 (2011).

[10] J. M. Dahlström, T. Carette, and E. Lindroth, Phys. Rev. A 86, 061402 (2012).

[11] M. Ivanov and O. Smirnova, Phys. Rev. Lett. 107, 213605 (2011).

[12] C.-H. Zhang and U. Thumm, Phys. Rev. A 82, 043405 (2010).

[13] S. Nagele, R. Pazourek, J. Feist, K. Doblhoff-Dier, C. Lemell, K. Tökési, and J. Burgdörfer, J. Phys. B 44, 081001 (2011).

[14] S. Nagele, R. Pazourek, J. Feist, and J. Burgdörfer, Phys. Rev. A 85, 033401 (2012).

[15] R. Pazourek, J. Feist, S. Nagele, and J. Burgdörfer, Phys. Rev. Lett. 108, 163001 (2012).

[16] M. Y. Amusia, Atomic Photoeffect (Plenum Press, New York, 1990).

[17] M. Y. Amusia and A. S. Kheifets, Phys. Lett. A 89, 437 (1982).

[18] K. T. Taylor, J. Phys. B 10, L699 (1977).

[19] L. V. Chernysheva, N. A. Cherepkov, and V. Radojevic, Comp. Phys. Comm. 11, 57 (1976).

[20] L. V. Chernysheva, N. A. Cherepkov, and V. Radojevic, Comp. Phys. Comm. 18, 87 (1979).

[21] U. Fano, Phys. Rev. A 32, 617 (1985).

[22] M. I. Amusia and L. V. Chernysheva, Computation of Atomic Processes: A Handbook for the ATOM Programs (Institute of Physics, Bristol, UK, 1997).

[23] J. Bizau and F. Wuilleumier, J. Electron Spectrosc. Relat. Phenom. 71, 205 (1995).

[24] Y. Ralchenko, A. E. Kramida, J. Reader, and NIST ASD Team, NIST Atomic Spectra Database (version 3.1.5) (National
Institute of Standards and Technology, Gaithersburg, MD, 2011) [http://physics.nist.gov/asd].

[25] M. Y. Amus'ya and A. S. Kheifets, Zh. Eksp. Teor. Fiz. 86, 1217 (1984) [Sov. Phys. JETP 59, 710 (1984)].

[26] L. Rosenenberg, Phys. Rev. A 52, 3824 (1995).

[27] B. Möbus et al., Phys. Rev. A 47, 3888 (1993).

[28] J. Samson and W. Stolte, J. Electr. Spectr. Relat. Phenom. 123, 265 (2002).

[29] U. Fano and J. W. Cooper, Rev. Mod. Phys. 40, 441 (1968).

[30] V. S. Yakovlev, J. Gagnon, N. Karpowicz, and F. Krausz, Phys. Rev. Lett. 105, 073001 (2010).

[31] R. G. Newton, Scattering Theory of Waves and Particles (Springer-verlag, New York, 1982).

[32] J. M. Dahlström (private communication).

[33] T. Carette, J. M. Dahlström, L. Argenti, and E. Lindroth, Phys. Rev. A 87, 023420 (2013).

[34] A. Ehresmann, F. Vollweiler, H. Schmoranzer, V. L. Sukhorukov, B. M. Lagutin, I. D. Petrov, G. Mentzel, and K. H. Schartner, J. Phys. B 27, 1489 (1994).

[35] S. Aksela, H. Aksela, M. Levasalmi, K. H. Tan, and G. M. Bancroft, Phys. Rev. A 36, 3449 (1987).

[36] Z. Altun and V. Radojevic, J. Phys. B 25, 3757 (1992).

[37] V. Schmidt, Rep. Prog. Phys. 55, 1483 (1992).

[38] U. Becker, D. Szostak, H. G. Kerkhoff, M. Kupsch, B. Langer, R. Wehlitz, A. Yagishita, and T. Hayaishi, Phys. Rev. A 39, 3902 (1989).

[39] A. Fahlman, M. O. Krause, T. A. Carlson, and A. Svensson, Phys. Rev. A 30, 812 (1984).

[40] M. Kutzner, V. Radojević, and H. P. Kelly, Phys. Rev. A 40, 5052 (1989).

[41] B. Zimmermann, G. Snell, B. Schmidtke, J. Viefhaus, N. A. Cherepkov, B. Langer, M. Drescher, N. Müller, U. Heinzmann, and U. Becker, Phys. Rev. A 64, 062501 (2001). 